\title{
WJU special edition: surgical and medical management of stones
}

\author{
Olivier Traxer
}

Published online: 21 January 2015

(C) Springer-Verlag Berlin Heidelberg 2015

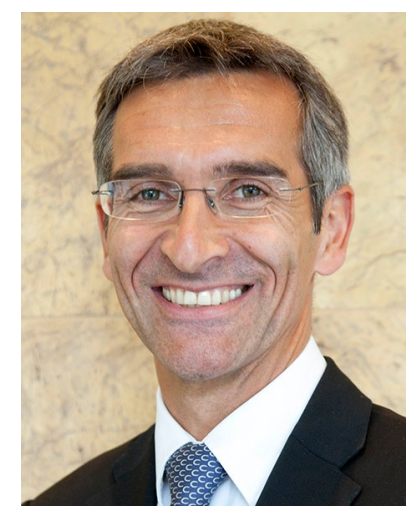

The development of urology over the years has been advancing along with that of stone disease. The management of patients affected with urolithiasis remains a major public health issue worldwide with a constant increase in its prevalence and incidence. Over the last three decades, the technological innovations have significantly reduced complications of open surgery while optimizing stone-free rate. Moreover, understanding the mechanisms of stone formation has allowed to identify lifestyle changes and specific drugs which should be adopted for reducing the risk of stone recurrence.

Nowadays, it is no longer possible to separate surgical treatment from medical management of urolithiasis. Indeed, all the clinicians approaching patients with urinary stones need to know not only all the surgical techniques available and their indications, but also they should offer them a metabolic evaluation and be able to manage the medical aspects of urolithiasis.

\section{O. Traxer $(\bowtie)$}

Head of the Endourological and Stone Unit, Urology Department Hôpital Tenon, Université Pierre et Marie CURIE Paris 6, Paris, France

e-mail: olivier.traxer@tnn.aphp.fr
This special edition of the World Journal of Urology sought to make an update on the medical and surgical management of stone disease.

The first six articles are focused on the medical aspects of urolithiasis.

Firstly, Michel Daudon, who is one of the biggest expert of the biologic mechanisms of stone formation, illustrated a morpho-constitutional classification of urinary stones underlining all the medical and environmental factors correlated with each type and sub-type of stone.

Secondly, Luc Valiquette and Julien Letendre explained how to interpret metabolic evaluation of stone patients and clarify what any urologist should know about this issue in 2015.

Thirdly, Margaret Pearle described all the links between diet and kidney stones.

Fourthly, Jean-Philippe Haymann reported all those rare diseases which can be revealed by the discovery of urinary stones.

In addition, since radiology is essential for the evaluation of urinary stones, I asked to Raphaëlle Renard-Penna to describe the developments of imaging techniques in this field. Finally, Palle Osther explained the medical treatment of acute renal colic, which is still the most common symptom of urinary stones, and its evolution over the last years focusing also on the indication for medical expulsive therapy (MET).

The second part of this "special issue" is purely centered on all the different treatment modalities.

Thomas Knoll provided a focus on extracorporeal lithotripsy, although this option seems to have lost a bit of appeal among the urological community compared with percutaneous nephrolithotomy (PCNL) and to the recent spread of ureterorenoscopy (URS). 
In addition, Jean de la Rosette summarized the results of the international survey of CROES (Clinical Research Office of Endourological Society) on the PCNL, and Mahesh Desai made a specific point on the miniaturization of technology and equipment in the field of percutaneous approach.

Finally, three articles are dedicated to the youngest, most innovative and most promising minimally invasive technique: the flexible ureterorenoscopy with laser fragmentation of the stone (f-URS).

Glenn Preminger exposed all the disposable equipment necessary for f-URS, while Michael Grasso described the different ureteroscopes and lasers available on the market.
In conclusion, Guido Giusti evaluated the state of the art of f-URS when applied in challenging cases and with particular indications.

I guess this "special issue" will fascinate the readers, as the management of stone disease patients does with me in my daily clinical practice.

A special thanks goes to all the authors, who really represent leading experts in this field, for their kind contribution to this special edition of the journal.

There is no doubt that this special issue of the World Journal of Urology will remain for a long time a reference for anyone who is interested in endourology and in the medical and surgical management of urinary stones. 